\title{
Fabrication and Performance of Selectively Oxidized Vertical-Cavity Lasers
}

\author{
Kent D. Choquette, K. L. Lear, R. P. Schneider, Jr., K. M. Geib, J. J. Figiel, and Robert Hull
}

\begin{abstract}
We report the high yield fabrication and reproducible performance of selectively oxidized vertical-cavity surface emitting lasers. We show that linear oxidation rates of AlGaAs without an induction period allows reproducible fabrication of buried oxide current apertures within monolithic distributed Bragg reflectors. The oxide layers do not induce obvious crystalline defects, and continuous wave operation in excess of $650 \mathrm{~h}$ has been obtained. The high yield fabrication enables relatively high laser performance over a wide wavelength span. We observe submilliamp threshold currents over a wavelength range of up to $75 \mathrm{~nm}$, and power conversion efficiencies at $1 \mathrm{~mW}$ output power of greater than $20 \%$ over a $50-\mathrm{nm}$ wavelength range.
\end{abstract}

$\mathbf{W}$ ET OXIDATION of AlGaAs [1], [2] has been successfully employed in the fabrication of edge emitting lasers, [3] and has recently been applied to various verticalcavity surface emitting laser (VCSEL) diode structures [4]-[9]. For example, VCSEL's incorporating GaAs-Al-oxide layers for high-index contrast distributed Bragg reflector (DBR) mirrors have been reported [4]. Partial oxidation of AlAs layers in the DBR mirror stack has been pursued to form a highindex cladding around the laser cavity [5]. Finally, using oxide layers to form current apertures under hybrid dielectric DBR's [6] or monolithic semiconductor DBR's [7] has also been demonstrated. The latter oxide-confined lasers have exhibited the highest power conversion efficiency $(>50 \%)$, [8] lowest threshold current density $\left(90 \mathrm{~A} / \mathrm{cm}^{2}\right.$ per quantum well), [9] and lowest threshold voltage ( $50 \mathrm{mV}$ above photon energy) [7] reported to date for VCSEL's. In this letter we report on the high-yield fabrication and reproducible performance of monolithic selectively oxidized VCSEL's. As opposed to record operation, we show that samples of large arrays of these lasers also exhibit relatively high performance levels over a wide lasing wavelength span that may be attractive for emerging VCSEL applications.

Fig. 1 is a schematic of our top-emitting VCSEL, which employs selective oxidation to produce buried oxide current apertures [7]. This oxide-confined VCSEL has several advantages. First, in this monolithic structure we fully exploit low resistance p-type DBR designs (such as parabolic heterointer-

Manuscript received June 23, 1995; revised July 24, 1995. The work at Sandia National Laboratories was supported in part by the United States DOE under contract No. DE-AC04-94AL85000.

K. D. Choquette, K. L. Lear, R. P. Schneider, Jr., K. M. Geib, and J. J. Figiel are with the Photonics Research Department, Sandia National Laboratories, Albuquerque, NM 87185 USA.

R. Hull is with the Department of Materials Science, University of Virginia, Charlottesville, VA 22903 USA.

IEEE Log Number 9414875.

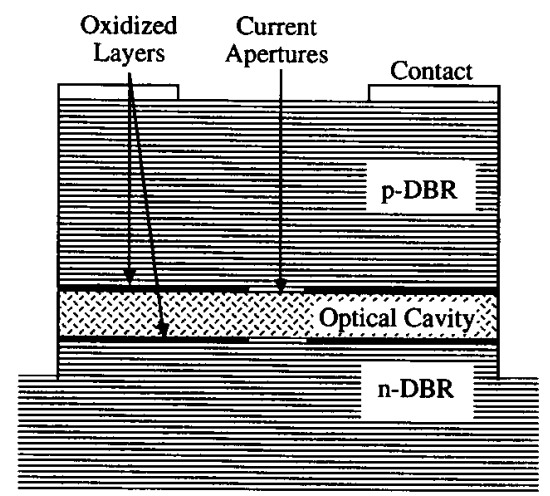

Fig. 1. Sketch of an oxide-confined VCSEL showing oxidized layers and the resulting current apertures on each side of the optical cavity.

face grading [10] and C-doping [11]) in utilizing the entire top mirror to conduct current into the active region. Thus, current crowding effects of thin intracavity contact layers [6] or ion implantation damage in the top DBR are both avoided. The current apertures immediately surrounding the optical cavity also eliminate sidewall nonradiative recombination present in etched air-post VCSEL's [12], and minimize lateral current spreading to outside of the laser cavity. Finally, the smaller refractive index of the Al-oxide layer [13] also induces index-guided optical confinement [14], [15], but in a planar VCSEL configuration amenable to efficient current flow and heat extraction. Comparing oxide-confined VCSEL's to ion implantation-defined VCSEL's, the enhanced electrical and optical confinement of the former produces reduced threshold current/voltage and the concomitant parasitic ohmic heating [9]. In the following, we describe the properties of $980 \mathrm{~nm}$ VCSEL's, although similar 850 and $650 \mathrm{~nm}$ [16] oxideconfined lasers have also been characterized.

The two-inch diameter VCSEL wafers are grown by metalorganic vapor phase epitaxy on a rotating susceptor, and have three InGaAs quantum wells in the active region. The monolithic DBR mirrors consist of $\mathrm{GaAs}-\mathrm{Al}_{0.92} \mathrm{Ga}_{0.08} \mathrm{As}$ layers with parabolic heterointerface grading. The low index layers adjacent to the optical cavity are adjusted to $\mathrm{Al}_{0.98} \mathrm{Ga}_{0.02} \mathrm{As}$ to increase their oxidation rates relative to the other DBR layers [7]. Fabrication of oxide-confined VCSEL's begins with the lift-off deposition of a top Ti-Pt-Au contact. A nitride mask is then deposited and patterned to encapsulate the metal contact and form a mesa etch mask. Reactive ion etching with $\mathrm{BCl}_{3}-\mathrm{Cl}_{2}$ is used to define the mesas and expose the sidewalls for oxidation. The samples are oxidized at $450{ }^{\circ} \mathrm{C}$ under flowing nitrogen, which is bubbled through water at 


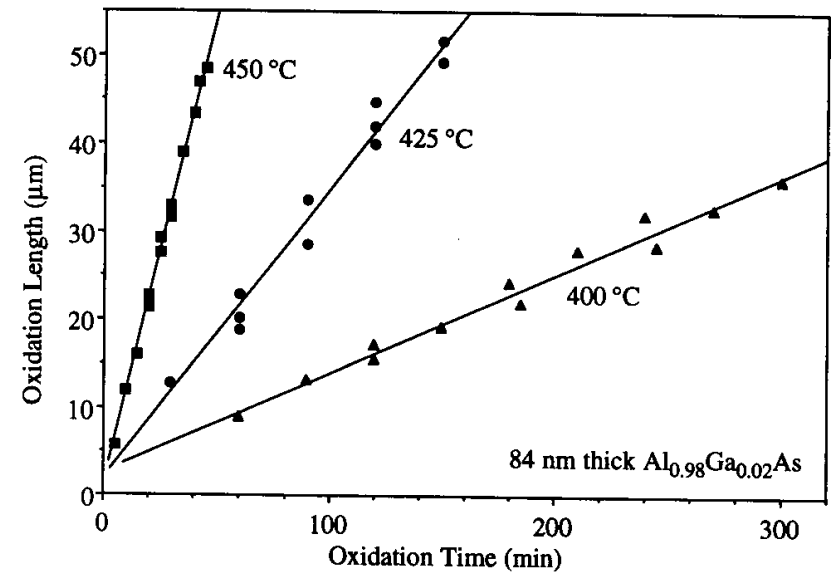

Fig. 2. Lateral extent of oxidation at 400,425 , and $450^{\circ} \mathrm{C}$ for a 84 -nm-thick $\mathrm{Al}_{0.98} \mathrm{Ga}_{0.02} \mathrm{As}$ layer contained within a distributed Bragg reflector, showing linear oxidation rates without induction periods.

$85^{\circ} \mathrm{C}$. After oxidation the nitride mask is removed and a backside $\mathrm{Ge}-\mathrm{Au}-\mathrm{Ni}-\mathrm{Au}$ contact is deposited and annealed. In this work we fabricate repeating rows of square mesas that vary in size from $48-90 \mu \mathrm{m}$ on a side. Oxidation conditions are selected to give an oxide penetration length of $24 \mu \mathrm{m}$ to "pinch off" the smallest mesa, thus producing arrays of VCSEL's with current apertures of $2 \times 2 \mu \mathrm{m}$ and larger. For each wafer examined, multiple samples are processed under similar oxidation conditions, where each sample extends from the wafer center to the edge.

To develop reproducible oxidation processes, test samples have been oxidized using different $\mathrm{Al}$ compositions, furnace temperatures and oxidation times. The extent of the lateral oxidation of a buried 84-nm-thick $\mathrm{Al}_{0.98} \mathrm{Ga}_{0.02} \mathrm{As}$ layer within a DBR mirror is plotted in Fig. 2. Fig. 2 shows that the lateral oxidation has a temperature dependent linear oxidation rate without an induction time preceding the onset of oxidation. Hence, reproducible fabrication of oxide apertures can be achieved on similar samples from a given wafer. However, the oxidation rate of $\mathrm{Al}_{x} \mathrm{Ga}_{1-x} \mathrm{As}$ is very sensitive to $\mathrm{Al}$ composition, where $x$ varying from $1-0.82$ changes the relative oxidation rate by more than two orders of magnitude [7]. Thus, the scatter of the oxidation rates in Fig. 2 may be partially related to small composition variations between the test samples. For example, near the edge of a wafer the oxidation rate is consistently found to monotonically decrease, presumably reflecting a slight increase of the $\mathrm{Ga}$ content arising from a growth edge effect. In addition, nominally identical wafers may have oxidation rates (at the center) that differ by $\approx 10 \%$. Therefore, stringent compositional control may be necessary for wafer scale manufacture of uniformly sized oxide apertures.

A cross-section transmission electron micrograph of a selectively oxidized VCSEL is shown in Fig. 3. The rounded terminus of the oxide layer in the top mirror is denoted at the vertical arrow in Fig. 3; the unoxidized region beyond this point corresponds to the interior of the current aperture which defines the laser cavity. In Fig. 3, and all other samples that have been examined by transmission electron microscopy, no dislocations or other crystalline defects are apparent along

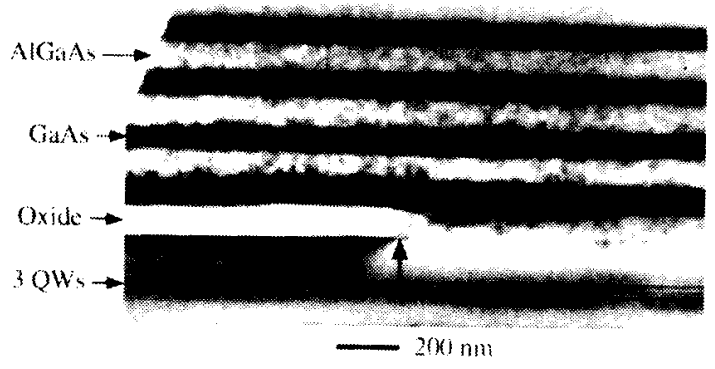

Fig. 3. Cross-section transmission electron micrograph $(g=(311)$ bright field image) of a selectively oxidized VCSEL showing the oxide layer above the quantum-well (QW) active region. The dark (light) layers in the DBR mirror are GaAs (AlGaAs). The terminus of the buried oxide layer, which forms the perimeter of the current aperture, is at the vertical arrow.

the oxide/semiconductor interface or near the oxidation terminus. Hence, the formation of the oxide layer does not induce obvious defects which would lead to degraded VCSEL operation or lifetime. This is consistent with our observation of approximately $98 \%$ yield of all oxide-confined VCSEL's tested to date. Moreover, the first lifetime experiment from a randomly chosen VCSEL has shown unchanged characteristics during $>650$ hours of $\mathrm{CW}$ operation at room temperature ( $7 \mathrm{~mA}$ constant current producing $1.1 \mathrm{~mW}$ output light) on a probe station.

In Fig. 4, we plot the threshold current, $I_{\text {th }}$, of VCSEL's from two wafers grown more than a month apart with the same epitaxial structure, which utilizes relatively high reffectivity top mirrors. The data points in Fig. 4 correspond to lasers that are spatially separated by $1 \mathrm{~mm}$, showing that both $I_{\mathrm{th}}$ and threshold wavelength vary radially, that latter due to thickness nonuniformity of the VCSEL wafers. The $I_{\mathrm{th}}$ wavelength variation arises from the spectral mismatch between the cavity resonance and the laser gain. The minimum $I_{\text {th }}$ occurs at $\approx 970 \mathrm{~nm}$, corresponding to the optimal overlap of cavity resonance/peak gain. Notice $I_{\mathrm{th}}$ around this wavelength are relatively insensitive to the lasing wavelength. Specifically over the wavelength span of $945-980 \mathrm{~nm}$, the average threshold current is $292 \mu \mathrm{A} \pm 6.5 \%$ for $5 \times 5-\mu \mathrm{m}$ VCSEL's in wafer XF114, and is $360 \mu \mathrm{A} \pm 5.3 \%$ for $7 \times$ $7-\mu \mathrm{m}$ VCSEL's in wafer XF225. For comparison, implanted VCSEL's (15 $\mu \mathrm{m}$ diameter) fabricated from wafer XF114 have an average threshold current of $4.4 \mathrm{~mA} \pm 10 \%$ over the same wavelength span of 945-980 $\mathrm{nm}$. All of the oxideconfined VCSEL's in Fig. 4 have threshold currents less than $1 \mathrm{~mA}$, corresponding to an operating wavelength span of up to $75 \mathrm{~nm}$. The maximum output power for the VCSEL's in Fig. 4 vary from $0.5-1.5 \mathrm{~mW}$, in spite of the relatively high output coupler reflectivity. Fig. 4 illustrates that submilliamp $I_{\mathrm{th}}$ can be reproducibly obtained from oxide-confined VCSEL's.

Fig. 5 depicts the power conversion (wall plug) efficiency obtained at 1-mW output power from VCSEL's fabricated from two other wafers. The lasers in Fig. 5 have reduced output coupler reflectivity where wafer XF130C has a single current aperture in the p-type DBR, and wafer XF130B has double apertures, as depicted in Fig. 1. For these wafers the highest efficiency again occurs at $\approx 970 \mathrm{~nm}$. The highest efficiency measured was $30 \%$, where the maximum typically 


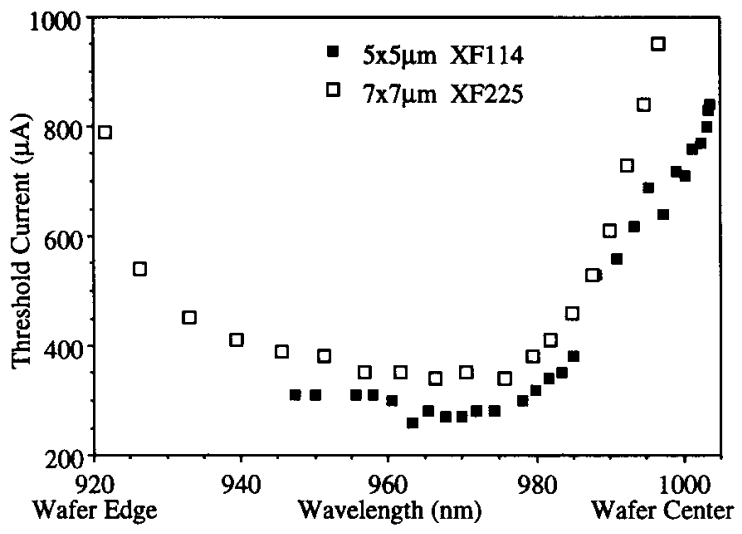

Fig. 4. Threshold current versus threshold wavelength at room temperature for oxide-confined VCSEL's from two wafers. The lasers in wafer XF114 $(\mathrm{XF} 225)$ have $5 \times 5-\mu \mathrm{m}(7 \times 7-\mu \mathrm{m})$ sized current apertures.

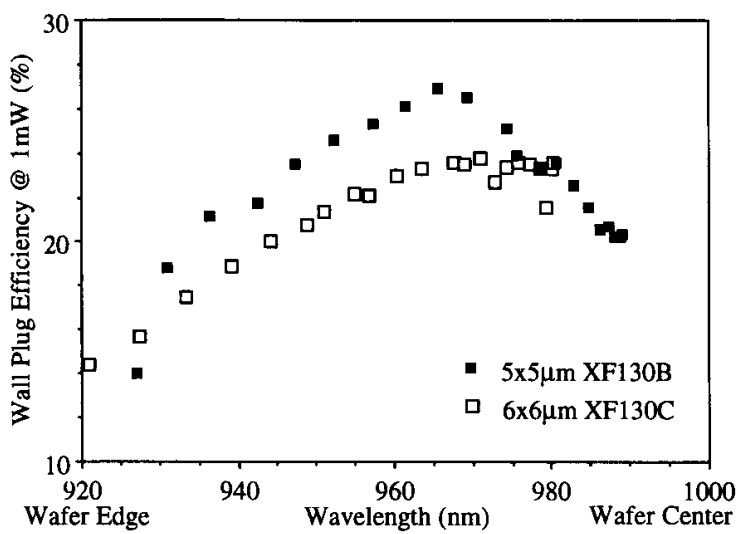

Fig. 5. Wall-plug efficiency at 1-mW output power at room temperature for oxide-confined VCSEL's. The lasers in wafer XF130B have a single $5 \times$ $5-\mu \mathrm{m}$ sized current aperture, while VCSEL's in wafer XF130C have two $6 \times$ $6-\mu \mathrm{m}$-sized current apertures surrounding the optical cavity.

occured between 1-3 $\mathrm{mW}$ output power. As shown in Fig. 5 at $1-\mathrm{mW}$ output power, greater than $20 \%$ efficiency is obtained over more than a 50 -nm wavelength range for wafer XF130B, and similar results are found from wafer XF130C (note the center resonance wavelength of this wafer is shorter). Over this wavelength range $I_{\text {th }}$ varies from $0.8-1.4 \mathrm{~mA}$ for VCSEL's from both wafers. Since $1 \mathrm{~mW}$ output is appropriate for many VCSEL applications, achieving high efficiency at this power is desirable. Maintaining wavelength insensitive $I_{\text {th }}$ and wall plug efficiency will impart higher yield to specification for VCSEL arrays in the presence of wafer nonuniformity.

In conclusion, monolithic oxide-confined VCSEL's not only demonstrate benchmark performance records, they also exhibit reproducible and wavelength insensitive performance levels which are attractive for potential applications. We have developed a reproducible and high yield selective oxidation process for fabrication of robust oxide-confined VCSEL's. Moreover, selectively oxidized VCSEL's have been fabricated at emission wavelengths of 980,850 , and $650 \mathrm{~nm}$, which indicates the universality of this VCSEL structure. High-performance oxide- confined VCSEL's, appropriate for a variety of wavelengths, should benefit emerging applications and markets considered for VCSEL's.

\section{ACKNOWLEDGMENT}

The authors would like to thank J. Walker of FEI Europe, Inc. for assistance with focused ion beam preparation of the TEM samples.

\section{REFERENCES}

[1] W. T. Tsang, "Self-terminating thermal oxidation of AlAs epilayers grown on GaAs by molecular beam epitaxy," Appl. Phys. Lett., vol. 33, pp. $426-429,1978$.

[2] J. M. Dallesasse, N. Holonyak, Jr., A. R. Sugg, T. A. Richard, and $\mathrm{N}$. El-Zein, "Hydrolyzation oxidation of $\mathrm{Al}_{x} \mathrm{Ga}_{1-x} \mathrm{As}-\mathrm{AlAs}-\mathrm{GaAs}$ quantum well heterostructures and superlattices," Appl. Phys. Lett., vol. 57, pp. 2844-2846, 1990

[3] J. M. Dallesasse and N. Holonyak, Jr., "Native-oxide stripe-geometry $\mathrm{Al}_{x} \mathrm{Ga}_{1-x} \mathrm{As}-\mathrm{GaAs}$ quantum well heterostructure lasers," Appl. Phys. Lett., vol. 58, pp. 394-396, 1991.

[4] M. H. MacDougal, P. D. Dapkus, V. Pudikov, H. Zhao, and G. M. Yang, "Ultralow threshold current vertical-cavity surface emitting lasers with AlAs-oxide-GaAs distributed Bragg reflectors," IEEE Photon. Technol. Lett., vol. 7, pp. 229-231, 1995.

[5] Y. Hayashi, T. Mukaihara, N. Hatori, N. Ohnoki, A. Matsutani, F. Koyama, and K. Iga, "Record low threshold index-guided InGaAs/AlGaAs vertical-cavity surface emitting laser with a native oxide confinement structure," Electron. Lett., vol. 31, pp. 560-561, 1995.

[6] D. L. Huffaker, D. G. Deppe, K. Kumar, and T. J. Rogers, "Native-oxide defined ring contact for low threshold vertical-cavity lasers," Appl. Phys. Lett., vol. 65, pp. 97-99, 1994.

[7] K. D. Choquette, R. P. Schneider, Jr., K. L. Lear, and K. M. Geib, "Low threshold voltage vertical-cavity lasers fabricated by selective oxidation," Electron. Lett., vol. 30, pp. 2043-2044, 1994.

[8] K. L. Lear, K. D. Choquette, R. P. Schneider, Jr., S. P. Kilcoyne, and K. M. Geib, "Selectively oxidized vertical-cavity surface emitting lasers with $50 \%$ power conversion efficiency," Electron. Lett., vol. 31, pp. 208-209, 1995.

[9] K. D. Choquette, K. L. Lear, R. P. Schneider, Jr., and K. M. Geib, "Cavity characteristics of selectively oxidized vertical-cavity lasers," Appl. Phys. Lett., vol. 66, pp. 3413-3415, 1995.

[10] R. P. Schneider, Jr., J. A. Lott, M. Hagerott Crawford, and K. D. Choquette, "Epitaxial design and performance of AlGaInP red (650-690 nm) VCSEL's," Inter. J. High Speed Electron. and Syst., vol. 5, pp. 625-666, 1994

[11] K. L. Lear, R. P. Schneider, Jr., K. D. Choquette, S. P. Kilcoyne, J. J. Figiel, and J. C. Zolper, "Vertical-cavity surface emitting lasers with $21 \%$ efficiency by metalorganic vapor phase epitaxy," IEEE Photon. Technol. Lett., vol. 6, pp. 1053-1055, 1994.

[12] K. D. Choquette, G. Hasnain, Y. H. Wang, J. D. Wynn, R. S. Freund, A. Y. Cho, and R. E. Leibenguth, "GaAs vertical-cavity surface emitting lasers fabricated by reactive ion etching," IEEE Photon. Technol. Lett., vol. 3, pp. 859-862, 1991.

[13] F. A. Kish, S. J. Caracci, N. Holonyak, Jr., J. M. Dallesasse, K. C. Hsieh, M. J. Ries, S. C. Smith, and R. D. Burnham, "Planar native-oxide indexguided $\mathrm{Al}_{x} \mathrm{Ga}_{1-x}$ As quantum well heterostructure lasers," Appl. Phys. Lett., vol. 59, pp. 1755-1757, 1991.

[14] D. L. Huffaker, J. Shin, and D. G. Deppe, "Lasing characteristics of low threshold microcavity lasers using half-wave spacer layers and lateral index confinement," Appl. Phys. Lett., vol. 66, pp. 1723-1725, 1995.

[15] K. L. Lear, K. D. Choquette, R. P. Schneider, Jr., and S. P. Kilcoyne, "Modal analysis of a small surface emitting laser with a selectively oxidized waveguide," Appl. Phys. Lett., vol. 66, pp. 2616-2618, 1995.

[16] K. D. Choquette, R. P. Schneider, Jr., M. H. Crawford, K. M. Geib, and J. J. Figiel, "Continuous wave operation of 640-660 nm selectively oxidized AlGaInP vertical-cavity lasers," Electron. Lett., vol. 31, pp. 1145-1146, 1995. 